\title{
P01-298
}

\section{ONLINE VICTIMIZATION, DEPRESSION AND ANXIETY AMONG ADOLESCENTS IN THE US}

B. Tynes ${ }^{1}$, M. Giang ${ }^{2}$

${ }^{1}$ Educational Psychology and Psychology, University of Illinois at Urbana-Champaign, Champaign, ${ }^{2}$ Psychological Studies in Education, University of California at Los Angeles, Los Angeles, USA

In the mid-1990s, the Internet was lauded for its potential to usher in an electronic global village free of race, gender and other physical indicators of difference. Recent research has shown, however, that many of the social ills that exist offline are recreated online. Adolescents experience these social ills as cyberbullying, online harassment, exposure to unwanted sexual solicitation/material and online racial prejudice. Scholars have tried to measure these experiences with questionnaires, but a standardized measure is needed in the field. In this presentation, we discuss the development of a comprehensive measure of online victimization developed to assess the nature, frequency and stress associated with these experiences. The Online Victimization Scale was administered online to 280 high school students in the US. A principal axis factor analysis was performed. The analysis yielded five factors: general victimization, sexual victimization, vicarious and group discrimination, stress, and individual discrimination. The factors accounted for $61 \%$ of the variance. Using the five factors, we examined the associations between online victimization and psychological adjustment. Online victimization was related to depression and anxiety over and above related offline victimization and stress measures. 